A NOVEL APPLICATION OF ERECTOR SPINAE PLANE BLOCK (ESP) PER I-OPERATIVE

ANALGESIA FOR PERCUTANEOUS NEPHROLITHOTOMY: CASE SERIES

Dr Sunil Kumar, Dr Parimala B, Dr Alka S Deo

\section{Introduction}

- PCNL is a surgical procedure commonly used to treat large kidney stones.

- Origin of pain: T10-T11 ( kidney)

T $10-\mathrm{L} 2$ ( ureter)

T $8-\mathrm{L} 2$ (incision)

- Various analgesia techniques:

Neuraxial blockades

Systemic opioids / IV analgesics

Paravertebral / Intercostal blocks

\section{Material \& Methods}

- Case series of 5 patients $\rightarrow$ PCNL Sx under GA + ESP

- Technique : GA $\rightarrow$ Prone position;

Level -T10 - T12 / Low frequency probe $18 \mathrm{G}$ touhy needle used.

- Drug used: $20 \mathrm{ml}$ of $\mathbf{0 . 3 7 5} \%$ Ropivacaine $+\mathbf{5 0} \mu \mathrm{g}$ of Dexmedetomidine

Haemodynamic variations (HR \& MAP) and additional requirement of intraoperative analgesics noted.

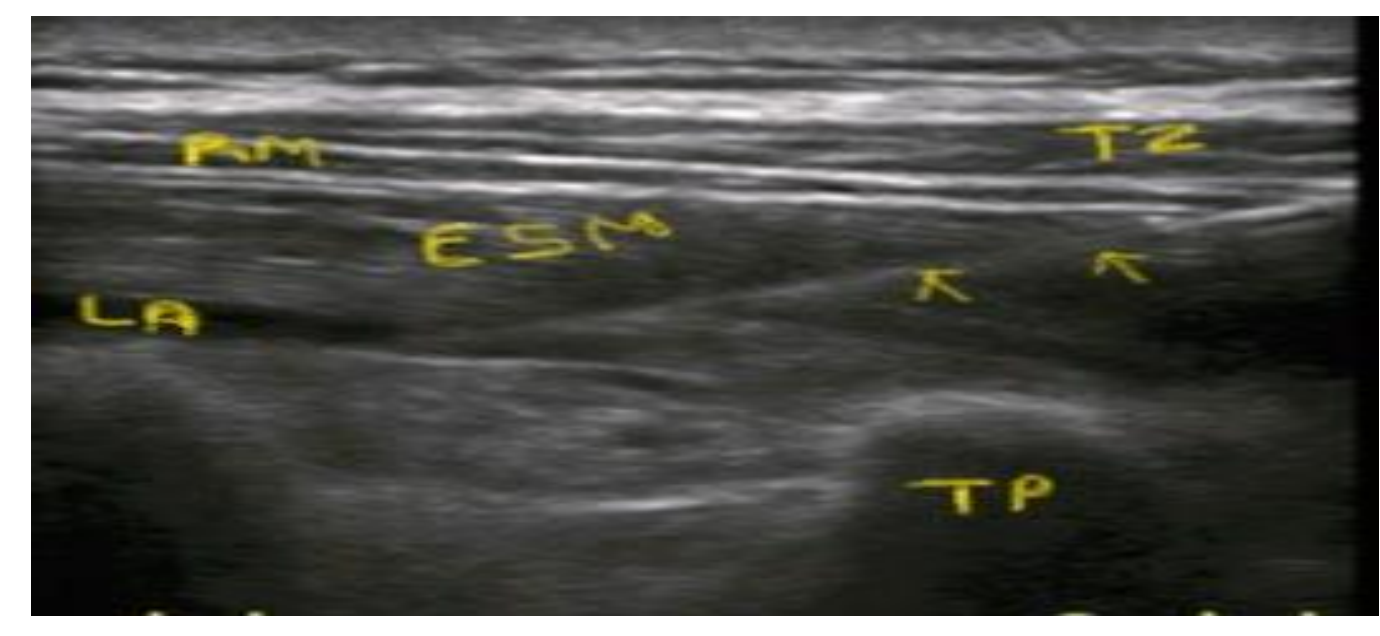

\section{Postop analgesia protocol}

- Postop pain was assessed by VAS score $2^{\text {nd }}$ hourly for 24 hours.

- Inj. Paracetamol $1 \mathrm{gm} 8$ th hourly iv irrespective of VAS score.

- Any patient with VAS score $\geq 4$ was treated with Inj. Tramadol 100mg iv.

\section{Data \& Results}

Intra-op findings:

No haemodynamic variations (HR and MAP) from baseline values $(>20 \%)$

$>$ None of the patients required additional analgesics in the intraoperative period.

Postop VAS and 24 hours analgesia:

\begin{tabular}{|c|c|c|}
\hline No of patients & VAS score & Additional analgesia (24 hrs) \\
\hline 4 & $<3$ & NIL \\
\hline 1 & $4($ at 16 hrs $)$ & Inj Tramadol 100mg i.v stat \\
\hline
\end{tabular}

\title{
Discussion and Conclusion:
}

ESP block is a interfacial plane block between transverse process and overriding erector spinae muscle.

Mechanism: LA drug diffuses through paravertebral space and acts on dorsal and ventral rami of spinal nerves + rami communicants. $\rightarrow$ Craniocaudal spread $\rightarrow$ Unilateral sensory and visceral analgesia.

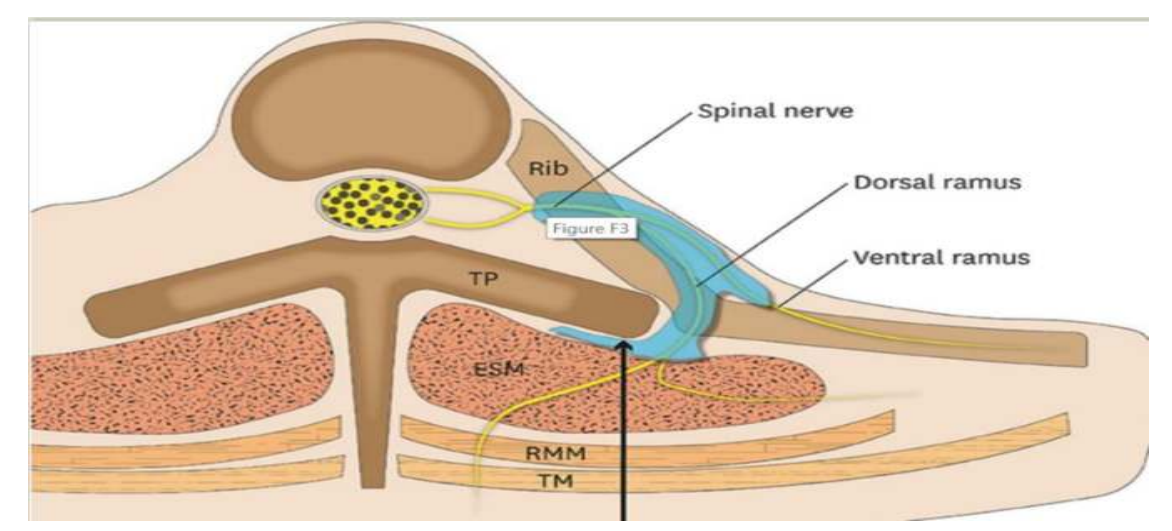

ESPB is a promising novel technique with expanding horizons in pain management; provides excellent perioperative analgesia for PCNL surgery.

Kim E, Kwon W, Oh S, Bang S. The Erector Spinae Plane Block for Postoperative Analgesia after Percutaneous Nephrolithotomy. Chinese medical journal. 2018 Aug 5;131(15):1877.

Forero M, Adhikary SD, Lopez H, Tsui C, Chin KJ. The erector spinae plane block: a novel analgesic technique in thoracic neuropathic pain. RAPM. 2016 Sep $1 ; 41(5): 621-7$. 